\title{
The symmetry energy at high density: new experimental results
}

\section{Wolfgang TRAUTMANN*}

GSI Helmholtzzentrum Darmstadt, Germany

E-mail: w.trautmannegsi.de

\section{QingFeng LI}

School of Science, Huzhou Teachers College, Huzhou 313000, P.R. China

E-mail: liqfer@gmail.com

\begin{abstract}
The nuclear equation of state of neutron-rich matter is essential for nuclear physics and astrophysics but still insufficiently known, in particular at high density. The symmetry energy representing the difference between the energy density of neutron matter and of symmetric matter influences properties of nuclei in their ground states and collective modes and governs properties of neutron stars, such as their internal structures, their radii and moments of inertia, their crustal vibration frequencies, and their cooling rates after formation in a supernova collapse.

Heavy-ion reactions are a unique tool for obtaining information on the symmetry energy in the laboratory. Nuclear structure and reaction observables have been successfully used to collect information on the equation-of-state for neutron-rich matter at densities below or near saturation. Observables sensitive to the early high-density phase reached in collisions at relativistic energies are expected to provide laboratory constraints for supra-saturation densities at which the uncertainties are largest. Significant results have already been obtained from isotopic effects measured for charged particle productions and collective flows but further work will be necessary to reach firm conclusions. Future opportunities can be expected from radioactive-beam facilities with neutron-rich beams of sufficient energy and intensity.
\end{abstract}

50th International Winter Meeting on Nuclear Physics

23-27 January 2012

Bormio, Italy

\footnotetext{
* Speaker.
} 


\section{Introduction}

Two years ago, at the time of the XLVIII International Winter Meeting on Nuclear Physics in Memoriam of Ileana Iori, the situation regarding the symmetry energy at high density as deduced from heavy-ion reaction data was partly unclear, perhaps even confusing [1]. Probes considered as very promising as, e.g., $\mathrm{K}^{+} / \mathrm{K}^{0}$ production ratios had turned out to be only weakly sensitive to the density dependence of the symmetry energy when measured double ratios were compared to transport calculations $[2,3]$, and results deduced from pion ratios were found to be highly model dependent $[4,5]$.

For the $\pi^{-} / \pi^{+}$yield ratios, variations of up to $20 \%$ for soft versus stiff parameterizations are expected. Unfortunately, the analysis of the data for the reaction ${ }^{197} \mathrm{Au}+{ }^{197} \mathrm{Au}$ measured by the FOPI Collaboration [6] led to even conflicting results, ranging from a rather stiff to a supersoft behavior of the symmetry energy $[4,5]$. Generally, the predictions with standard parameters were found to be too small [6]. Therefore, more extreme assumptions had to be made to reach the experimental values which, depending on the model, required either very stiff or very soft solutions $[2,4,5]$. Among them, the super-soft result has initiated a broad discussion of how it might be reconciled with observed properties of neutron stars $[4,7,8]$.

While new interpretations of the measured meson ratios have not appeared up to now, new experimental results regarding the density dependence of the symmetry energy below and above saturation have become available. A promising consensus seems to emerge from nuclear structure and reaction studies, probing nuclear matter below saturation and favoring a moderately soft behavior which may be characterized with a value $L \approx 70 \mathrm{MeV}$ for the parameter representing the slope of the symmetry energy as a function of density at saturation [9]. The corresponding thickness of the neutron skin of the ${ }^{208} \mathrm{~Pb}$ nucleus is $\Delta r_{n p} \approx 0.20 \mathrm{fm}$. The two quantities have been found to be highly correlated [10], and either one of them may, therefore, be used to express the result of a particular experimental or theoretical study.

Besides isotopic ratios of produced particles, also collective flows have been proposed as probes for studying the symmetry energy at supra-saturation densities [11, 12], among them the so-called differential neutron-proton flow which is the difference of the parameters describing the collective motion of free neutrons and protons weighted by their numbers [11]. Also the ratios or the differences of mean flow parameters of neutrons with respect to light charged particles have been found to be very useful. From the analysis of the existing data set for the reaction ${ }^{197} \mathrm{Au}+{ }^{197} \mathrm{Au}$ at $400 \mathrm{MeV} /$ nucleon $[13,14]$, the elliptic flow has emerged as an observable that depends sensitively on the density dependence of the symmetry energy $[15,16]$. The comparison of the measured flow parameters with the predictions of transport models favors a moderately soft to nearly linear behavior of the symmetry energy with density that is consistent with the sub-saturation results but incompatible with the super-soft behavior deduced from the pion data.

Important new information is expected to come from experiments, either in preparation for being soon conducted or already carried out with the analysis in progress, which will be briefly addressed. The following sections will be mainly devoted to new results contributing to the present knowledge of the $L$ parameter and to the recent analyses of the elliptic flow in ${ }^{197} \mathrm{Au}+{ }^{197} \mathrm{Au}$, including the study of parameter dependences in the model descriptions. 


\section{The slope at saturation}

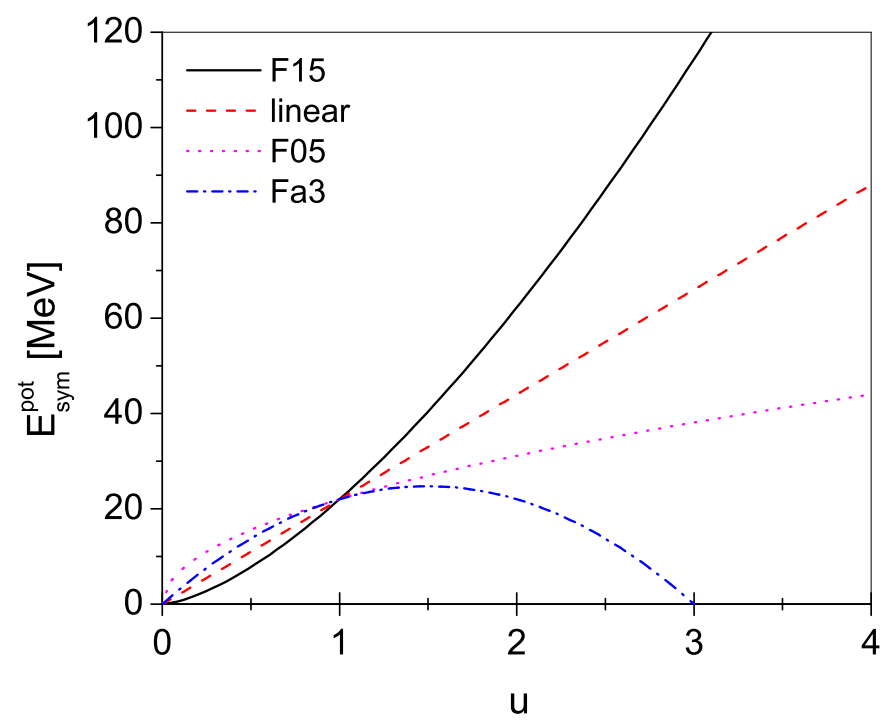

Figure 1: The symmetry potential energy of nucleons as a function of the reduced nuclear density $u=\rho / \rho_{0}$ using various density dependences. Power-law functions with $\gamma=1.5,1.0$., and 0.5 are indicated as F15, linear, and F05, respectively. In the super-soft case Fa3, the potential part is proportional to $u(3-u) / 2$ (from Ref. [18]).

The symmetry energy, i.e. the symmetry-term coefficient $E_{\text {sym }}$ in the usual quadratic expansion of the equation of state,

$$
E / A(\rho, \delta)=E / A(\rho, \delta=0)+E_{\mathrm{sym}}(\rho) \cdot \delta^{2}
$$

is the difference between the energies of symmetric matter $(\delta=0)$ and neutron matter $(\delta=1)$ with $\delta=\left(\rho_{n}-\rho_{p}\right) / \rho$ and $\rho_{n}, \rho_{p}$, and $\rho$ representing the neutron, proton, and total densities, respectively.

To be used in reaction models based on transport theory, often simplified descriptions of the composition-dependent part of the nuclear mean field are required. In the UrQMD [18, 19] used for the analysis of the elliptic flow in ${ }^{197} \mathrm{Au}+{ }^{197} \mathrm{Au}$, the density dependence of the potential part of the symmetry energy is described with the power-law coefficient $\gamma$, while a second parameter gives the strength at the saturation density $\rho_{0} \approx 0.16$ nucleons $/ \mathrm{fm}^{3}$,

$$
E_{\mathrm{sym}}=E_{\mathrm{sym}}^{\mathrm{pot}}+E_{\mathrm{sym}}^{\mathrm{kin}}=22 \mathrm{MeV} \cdot\left(\rho / \rho_{0}\right)^{\gamma}+12 \mathrm{MeV} \cdot\left(\rho / \rho_{0}\right)^{2 / 3},
$$

Examples of these parameterizations are given in Fig. 1. In the IBUU04 developed by the groups of $\mathrm{Li}$ and Chen $[20,21]$, the nuclear potential of Das et al. with explicit momentum dependence in the isoscalar and isovector parts is used [22]. The density dependence of the symmetry energy is characterized by a parameter $x$ appearing in the potential expressions. With $x=0$, the density dependence is similar to $\gamma=0.5$ and with $x=-1$ similar to $\gamma=1.3$, while $x=+1$ and $x=-2$ represent the super-soft and super-stiff cases, respectively. 
The density dependence of the symmetry energy is often expressed in the form of the parameter $L$ which is proportional to the derivative with respect to density at saturation,

$$
L=3 \rho_{0} \cdot d E_{\mathrm{sym}} / d \rho \mid \rho_{0} .
$$

Quite compatible results, with their uncertainties falling into the interval $20 \mathrm{MeV} \leq L \leq 100 \mathrm{MeV}$, have been obtained from experiments testing the strength of the symmetry term at densities near or below saturation. This includes isospin diffusion and neutron/proton yield ratios [23] as well as the isospin dependence of the isoscalar giant monopole resonance [24] or the strength of the pygmy resonance in neutron rich nuclei [25]. A presently most probable value is $L \approx 60-70 \mathrm{MeV}$, roughly corresponding to a power-law coefficient $\gamma=0.6$.

These results have found confirmation in very recent experiments. The isospin equilibration in $\mathrm{Sn}+\mathrm{Sn}$ collisions has been studied at an incident energy of $35 \mathrm{MeV} /$ nucleon by constructing the isospin diffusion ratios from the measured yields of the $A=7$ mirror nuclei ${ }^{7} \mathrm{Li}$ and ${ }^{7} \mathrm{Be}$ [26]. The results agree with the transport model predictions based on the symmetry-energy constraints previously established at $50 \mathrm{MeV} /$ nucleon [23]. The scattering of protons of several hundred $\mathrm{MeV}$ has been shown to be a promising new spectroscopic tool for studying the electric dipole strength in nuclei [27]. Using the correlation between the total electric dipole polarizability and the neutron skin [28], a skin thickness of $0.16 \pm 0.02$ fm for ${ }^{208} \mathrm{~Pb}$ has been deduced from these data. According to the correlation obtained for empirical mean-field interactions [10], it corresponds to a slope parameter $L \approx 40 \pm 25 \mathrm{MeV}$. It is very encouraging to find rather similar constraints being also deduced from very recent investigations and observations of neutron-star properties [29, 30, 31].

High expectations are connected with the PREX experiment aiming at measuring the parityviolating contribution to electron scattering at high energy [32]. Very little model dependence is involved in determining the neutron-skin thickness of ${ }^{208} \mathrm{~Pb}$ from the observed anisotropy [33]. Once completed, this experiment will provide a practically model-free access to the density dependence of the symmetry energy, obtained from probing nuclear matter at densities below saturation [34].

\section{FOPI-LAND elliptic-flow data}

Preliminary results from the reanalysis of the FOPI-LAND data on elliptic flow in ${ }^{197} \mathrm{Au}+$ ${ }^{197} \mathrm{Au}$ collisions at $400 \mathrm{MeV} /$ nucleon have been reported two years ago [1]. The data have been taken in experiments at GSI with the FOPI (Phase 1) and LAND detectors, then providing evidence for the squeeze-out of neutrons in this energy regime $[13,14]$. The motivation for returning to the existing data set has come from studies performed with the UrQMD transport code for this fairly neutron-rich system $(N / Z=1.49)$ which indicated a significant sensitivity of elliptic-flow to the assumptions made for the density dependence of the symmetry energy [15]. This is illustrated in Fig. 2, top panel, which shows the dependence of the elliptic flow parameter $v_{2}$ on the transverse momentum per nucleon, $p_{t} / A$, for the combined central and mid-peripheral collisions collected in this experiment. The parameter $v_{2}$ is the second-order coefficient of a Fourier decomposition of the azimuthal distributions measured with respect to the orientation of the reaction plane (see, e.g., Refs. $[15,35])$. The measured values are approximately reproduced by the UrQMD predictions. For hydrogens (not shown), the results for the stiff $(\gamma=1.5)$ and soft $(\gamma=0.5)$ density dependences are similar and close to the asy-stiff predictions for neutrons. For neutrons, however, the predictions 
are different by, on average, $20 \%$ for the main part of the $p_{t}$ interval (dashed lines) but seem to converge at high $p_{t}$ at which the yields become small and the statistical errors large.

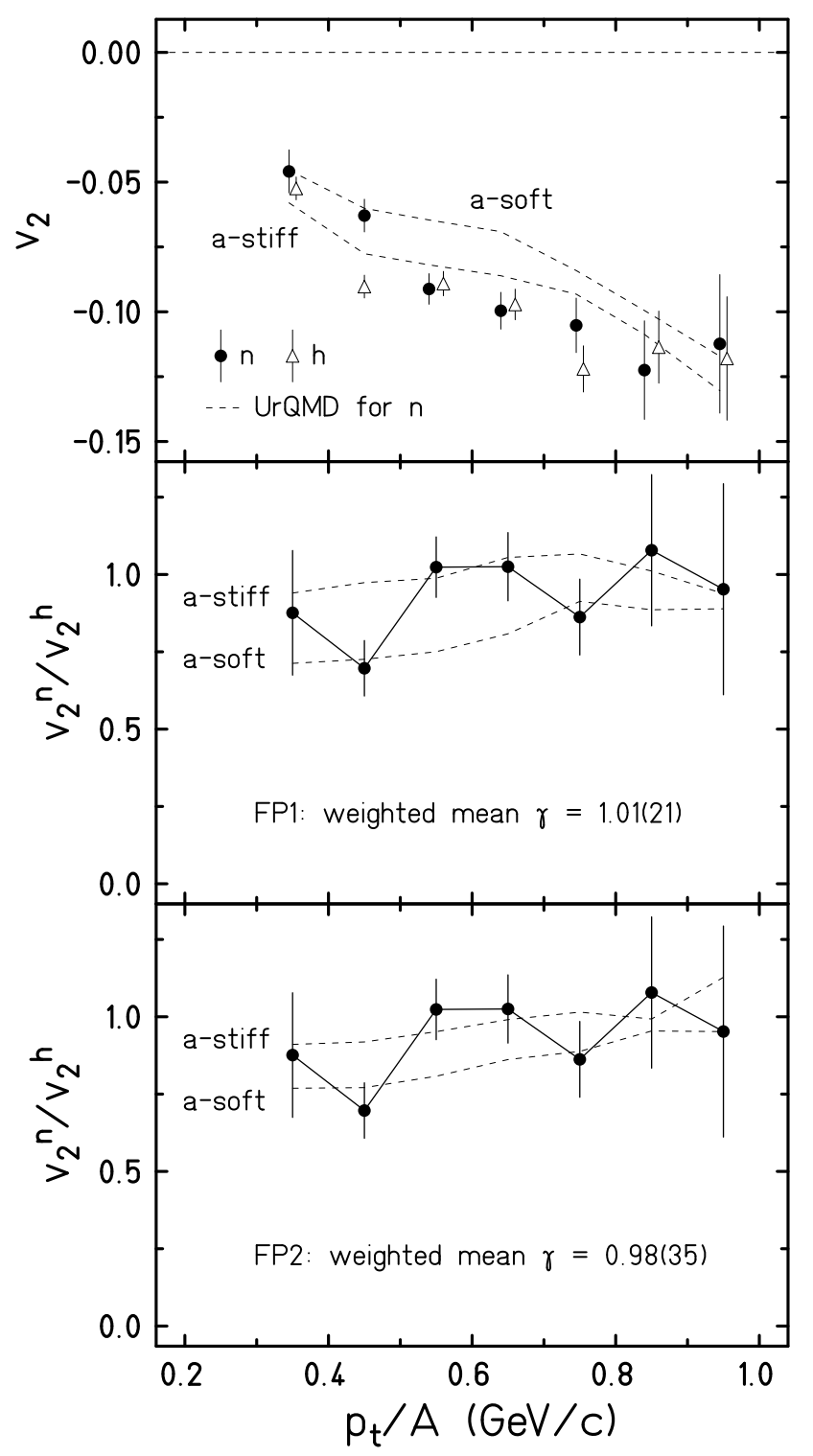

Figure 2: Elliptic flow parameters $v_{2}$ for neutrons (dots) and hydrogen isotopes (open triangles, top panel) and their ratio (lower panels) for moderately central $(b<7.5 \mathrm{fm})$ collisions of ${ }^{197} \mathrm{Au}+{ }^{197} \mathrm{Au}$ at 400 $\mathrm{MeV} /$ nucleon, integrated within the rapidity interval $0.25 \leq y / y_{p} \leq 0.75$, as a function of the transverse momentum per nucleon $p_{t} / A$. The symbols represent the experimental data. The UrQMD predictions for $\gamma=1.5$ (a-stiff) and $\gamma=0.5$ (a-soft) obtained with the FP1 parameterization for neutrons (top panel) and for the ratio (middle panel), and with the FP2 parameterization for the ratio (bottom panel) are given by the dashed lines. Note the suppressed zero of the abscissa (from Ref. [15]).

For the model comparison, ratios of the flow parameters of neutrons versus protons or versus $Z=1$ particles have been used in order to minimize systematic effects influencing the collective flows of neutrons and charged particles in similar ways. It also aids in the actual comparisons because uncertainties of how to match the impact-parameter intervals used in the calculations with 
the corresponding experimental event groups should cancel to first order. To demonstrate this property, the calculations were performed with two parameterizations of the momentum dependence of the elastic nucleon-nucleon cross section, labelled FP1 and FP2, which differ in their absolute predictions of $v_{2}$ at mid-rapidity by $\approx 40 \%$ for this reaction $[36,37]$.

The results for the ratio $v_{2}^{n} / v_{2}^{h}$, i.e. with respect to the integrated hydrogen yield, are shown in Fig. 2 (lower panels). Their sensitivity to the stiffness of the symmetry energy as predicted by the UrQMD is clearly visible. It is slightly smaller with the FP2 parameterization of the in-medium nucleon-nucleon cross section (bottom panel). The experimental ratios scatter within the intervals given by the calculations for $\gamma=0.5$ and 1.5 in similar ways, indicating that the effects of the cross section parameterization largely cancel in the ratios. Linear interpolations between the predictions, averaged over $0.3<p_{t} / A \leq 1.0 \mathrm{GeV} / \mathrm{c}$, yield practically identical results for the two cases.

The analysis was repeated for the squeeze-out ratios of neutrons with respect to free protons, and the dependence on impact parameter was studied. In consideration of the apparent systematic and experimental errors, a value $\gamma=0.9 \pm 0.4$ has been adopted as best representing the power-law exponent of the potential term resulting from the elliptic-flow analysis. It falls slightly below the $\gamma=1.0$ line shown in Fig. 1 but, with the quoted uncertainty, stretches over a wide interval from $\gamma=0.5$ halfway up to $\gamma=1.5$. The corresponding slope parameter is $L \approx 80 \pm 25 \mathrm{MeV}$.

A simple test of which densities are probed was finally made by reducing the coefficient of the potential term in the symmetry energy from its default value $22 \mathrm{MeV}$ to $18 \mathrm{MeV}$ (cf. Eq. 2.2). If mainly the strength of the symmetry energy below saturation is important, this modification will require a considerably softer density dependence in order to preserve a high level there. The opposite is expected if the differential flow is primarily determined by its strength at super-saturation density. The actual results were very similar, favoring neither one of the two cases but confirming that both, sub- and supra-saturation densities are probed with this observable [15].

\section{Medium and momentum dependent cross sections}

It is well known that the nucleon-nucleon cross sections will be significantly affected by the nuclear medium (see, e.g., Refs. [38, 39]). In the UrQMD model, the elastic in-medium cross sections are parameterized as the product of the free nucleon-nucleon elastic cross sections and an in-medium correction factor $F$ which is proportional to both the isospin-scalar density effect and the isospin-vector mass-splitting effect as discussed in Refs. [19, 37].

The corrections $F(u)$, depending on the relative density $u=\rho / \rho_{0}$, should, furthermore, be functions of the relative momentum $p_{N N}$ of the two colliding particles in the nucleon-nucleon center-of-mass system, formulated as

$$
F\left(u, p_{N N}\right)=\left\{\begin{array}{lr}
f_{0} & p_{N N}>1 \mathrm{GeV} / c \\
\frac{F(u)-f_{0}}{1+\left(p_{N N} / p_{0}\right)^{\kappa}}+f_{0} & p_{N N} \leq 1 \mathrm{GeV} / c .
\end{array}\right.
$$

Here, $f_{0}, p_{0}$ and $\kappa$ are parameters for which, again, several options exist as shown in the following table and illustrated in Fig. 3.

The standard option for UrQMD calculations is the density dependent correction $F(u)$ with the parameterization FU1 but other options have been found useful in special cases [37]. The 


\begin{tabular}{|l|c|c|c|}
\hline Set & $f_{0}$ & $p_{0}\left[\mathrm{GeV} \mathrm{c}^{-1}\right]$ & $\kappa$ \\
\hline \hline FP1 & 1 & 0.425 & 5 \\
FP2 & 1 & 0.225 & 3 \\
FP3 & 1 & 0.625 & 8 \\
\hline no $p_{N N}$ limit & $F(u)$ & & \\
\hline
\end{tabular}

Table 1: The three parameter sets FP1, FP2, and FP3 used for describing the momentum dependence of the correction factor $F$. The fourth case, without a $p_{N N}$ limit, is obtained by setting $f_{0}$ equal to $F(u)$ in Eq. (4.1).

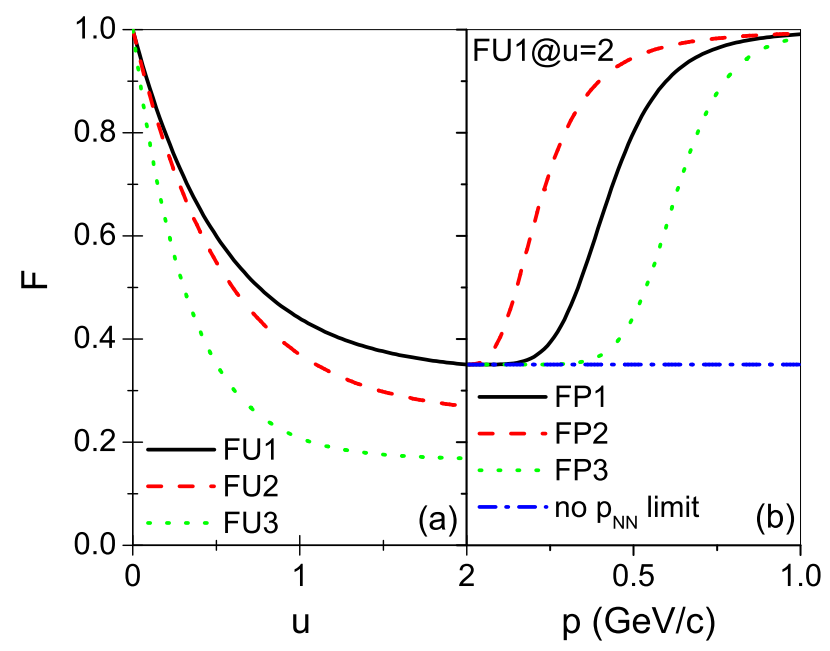

Figure 3: (a) Correction factor $F$ obtained with three parameterizations FU1, FU2, and FU3 for the density dependence and (b) the momentum dependence for the example of FU1 at $u=2$ obtained with the four options FP1, FP2, FP3, and "no p $_{N N}$ limit" (Eq. 4.1 with parameters given in Table 1).

restoration of the free elastic cross sections at high momentum is essential for reaching the observed magnitude of the elliptic flow. In the "no $p_{n n}$ limit", the predicted $v_{2}$ at mid-rapidity for the studied ${ }^{197} \mathrm{Au}+{ }^{197} \mathrm{Au}$ collisions at $400 \mathrm{MeV} /$ nucleon amounts to only half the measured value. With the FP1 or FP2 parameterizations, the predictions come close but still slightly underpredict or overpredict, respectively, the experimental value by $15 \%$ to $20 \%[15,37]$. The difference in absolute magnitude of nearly $40 \%$ is significant. It is, therefore, very encouraging to find the effect of the momentum dependence of the elastic cross sections to be cancelled out when flow ratios are studied (Fig. 2).

In a very recent analysis, Cozma has also investigated the influence of several parameters on the the elliptic flows of protons and neutrons using the Tübingen version of the QMD transport model [16]. They included, in particular, the choice of various forms of free and in-medium nucleon-nucleon cross sections. It was found that the effects of choosing different cross section parameterizations had almost an order-of-magnitude smaller effect on the difference between proton and neutron elliptic flows than variations of the density dependence of the symmetry energy. A super-soft behavior of the symmetry energy was confirmed to be excluded by the comparison with the flow data from the FOPI-LAND experiment [14]. 


\section{Conclusion and outlook}

The consensus emerging from studies near or below saturation, indicating a moderately soft density dependence of the symmetry energy, is very encouraging [9]. Astrophysical observations seem to support this result. However, more direct observables probing nuclear matter at higher densities will still be needed. It is, therefore, important to continue the investigation of yield ratios of isospin pairs of produced mesons and to explore their connection with the very first stages of the collision [2].

The squeeze-out data for ${ }^{197} \mathrm{Au}+{ }^{197} \mathrm{Au}$ collisions at $400 \mathrm{MeV} /$ nucleon indicate a moderately soft to linear behavior of the symmetry energy that is consistent with the density dependence deduced from heavy-ion reactions at lower energies and from nuclear structure experiments. Even though twice the saturation density can be reached in this reaction, the density regime that is actually probed needs to be determined more precisely. The statistical uncertainty of the existing FOPI-LAND data is larger than the systematic effects investigated so far. Highly improved results can thus be expected from a new experiment delivering a comprehensive data set with sufficiently high statistical accuracy. It will be interesting to see first results appearing from the recently completed measurements of the ASYEOS Collaboration at the GSI Laboratory [41].

In the long term, the continuation of this program with systems of larger asymmetry will be very promising and important. Radioactive beam facilities will enlarge the possibilities for using neutron-rich beams to be combined with isotopically enriched targets. More efficient detector setups will be needed to compensate the corresponding loss in luminosity. For the measurement of neutrons, the neutron detector NeuLAND proposed for experiments at FAIR will offer a highly improved detection efficiency in the energy range 100 to $400 \mathrm{MeV}$ [42]. It will permit extending the program also to reactions at lower energies for which significant mean-field effects are predicted for directed and elliptic flows [40].

Illuminating discussions with M.D. Cozma and H.H. Wolter are gratefully acknowledged.

\section{References}

[1] W. Trautmann, in proceedings of XLVIII International Winter Meeting on Nuclear Physics in Memoriam of Ileana Iori, POS (BORMIO2010) 061.

[2] G. Ferini et al., Nucl. Phys. A 762 (2005) 147; Phys. Rev. Lett. 97 (2006) 202301.

[3] X. Lopez et al., Phys. Rev. C 75 (2007) 011901(R).

[4] Zhigang Xiao et al., Phys. Rev. Lett. 102 (2009) 062502.

[5] Zhao-Qing Feng and Gen-Ming Jin, Phys. Lett. B 683 (2010) 140.

[6] W. Reisdorf et al., Nucl. Phys. A 781 (2007) 459.

[7] Bao-An Li et al., J. Phys. Conf. Ser. 312 (2011) 042006.

[8] De-Hua Wen, Bao-An Li, Lie-Wen Chen, Phys. Rev. Lett. 103 (2009) 211102.

[9] M.B. Tsang et al., preprint arXiv:1204.0466[nucl-ex] (2012).

[10] X. Roca-Maza, M. Centelles, X. Viñas, M. Warda, Phys. Rev. Lett. 106 (2011) 252501.

[11] Bao-An Li, Phys. Rev. Lett. 88 (2002) 192701.

[12] V. Greco et al., Phys. Lett. B 562 (2003) 215.

[13] Y. Leifels et al., Phys. Rev. Lett. 71 (1993) 963.

[14] D. Lambrecht et al., Z. Phys. A 350 (1994) 115. 
[15] P. Russotto et al., Phys. Lett. B 697 (2011) 471.

[16] M.D. Cozma, Phys. Lett. B 700 (2011) 139.

[17] C. Fuchs and H.H. Wolter, Eur. Phys. J. A 30 (2006) 5.

[18] Q. Li et al., J. Phys. G 31 (2005) 1359;

[19] Q. Li et al., J. Phys. G 32 (2006) 151; ibid. 32 (2006) 407.

[20] for a recent review, see Bao-An Li, Lie-Wen Chen, and Che Ming Ko, Phys. Rep. 464 (2008) 113.

[21] Bao-An Li and Lie-Wen Chen, Phys. Rev. C 72 (2005) 064611.

[22] C.B. Das, S. Das Gupta, C. Gale, Bao-An Li, Phys. Rev. C 67 (2003) 034611.

[23] M.B. Tsang et al., Phys. Rev. Lett. 102 (2009) 122701.

[24] T. Li et al., Phys. Rev. C 81 (2010) 034309.

[25] A. Klimkiewicz et al., Phys. Rev. C 76 (2007) 051603(R).

[26] Z.Y. Sun et al., Phys. Rev. C 82 (2010) 051603.

[27] A. Tamii et al., Phys. Rev. Lett. 107 (2011) 062502.

[28] P.-G. Reinhard and W. Nazarewicz, Phys. Rev. C 81 (2010) 051303(R).

[29] K. Hebeler, J.M. Lattimer, C.J. Pethick, and A. Schwenk, Phys. Rev. Lett. 105 (2010) 161102.

[30] A.W. Steiner, J.M. Lattimer, and E.F. Brown, ApJ. 722 (2010) 33.

[31] H. Sotani, K. Nakazato, K. Iida, K. Oyamatsu, preprint arXiv:1202.6242[astro-ph.HE] (2012).

[32] S. Abrahamyan et al., Phys. Rev. Lett. 108 (2012) 112502.

[33] C.J. Horowitz et al., Phys. Rev. C 85 (2012) 032501(R).

[34] K. Kumar, overview talk at this conference and contribution to this procedings.

[35] A. Andronic, J. Łukasik, W. Reisdorf, W. Trautmann, Eur. Phys. J. A 30 (2006) 31.

[36] Qingfeng Li, Caiwan Shen, M. Di Toro, Mod. Phys. Lett. A 25 (2010) 669.

[37] Qingfeng Li et al., Phys. Rev. C 83 (2011) 044617.

[38] G.Q. Li and R. Machleidt, Phys. Rev. C 48 (1993) 1702.

[39] G.Q. Li and R. Machleidt, Phys. Rev. C 49 (1994) 566.

[40] ChenChen Guo et al., Science China Physics, Mechanics \& Astronomy 55 (2012) 252.

[41] R.C. Lemmon et al., proposal for SIS experiment S394 (2009).

[42] NeuLAND Technical Design Report, submitted to FAIR (2011). 